\title{
Circular RNA Hsa_Circ_0091579 Serves as a Diagnostic and Prognostic Marker for Hepatocellular Carcinoma
}

\author{
Chenxing Zhang ${ }^{a} \quad$ Chenyue Zhang ${ }^{b}$ Jiamao Lin ${ }^{c}$ Haiyong Wang ${ }^{c}$ \\ aDepartment of Allergy and Immunology, Shanghai Children's Medical Center, Shanghai Jiao Tong \\ University School of Medicine, Shanghai, 'Department of Integrative Oncology, Fudan University \\ Shanghai Cancer Center, Shanghai, 'Department of Internal Medicine-Oncology, Shandong Cancer \\ Hospital and Institute, Shandong Cancer Hospital affiliated to Shandong University, Shandong \\ Academy of Medical Sciences, Jinan, China
}

\section{Key Words}

Hepatocellular carcinoma • Hsa_circ_0091579 • Diagnosis • Prognosis • Biomarker

\begin{abstract}
Background/Aims: An increasing number of studies have suggested that circular RNAs (circRNAs) have vital roles in carcinogenesis and tumor progression. However, the function of circRNAs in hepatocellular carcinoma (HCC) remains poorly characterized. Methods: We investigated the levels of circRNAs in patients with HCC to identify potential diagnostic biomarkers. We examined circRNA expression profiles in liver tumors and paired noncancerous liver tissues from three HCC patients with cancer thrombus using a circRNA microarray. Bioinformatics analysis was performed to find circRNAs with significantly altered expression levels between tumors and their paired non-tumor tissues. We confirmed our initial findings by quantitative reverse transcription-polymerase chain reaction (qRT-PCR). Receiver operating characteristic (ROC) curves were also applied to identify a candidate circRNA with the optimal specificity and sensitivity. Finally, X-tile software was adopted to calculate the most efficient cut-off value for hsa_circ_0091579 expression. Results: Microarray analysis identified 20 unique circRNAs that were differentially expressed between tumor and non-tumor tissues $(P<0.05)$. The expression of these 20 circRNAs was verified by qRT-PCR. The expression of hsa_circ_16245-1 and hsa_circ_0091579 mRNA was consistent with their levels as tested by the microarray. The ROC curves showed that both hsa_circ_16245-1 and hsa_circ_0091579 had favorable specificity and sensitivity. We further confirmed that hsa_circ_0091579 was significantly upregulated in HCC and its high expression was intimately associated with a worse overall survival in patients with HCC. Conclusion: Hsa_circ_0091579 may play a critical role in HCC progression and serve as a potential biomarker for the prognosis of patients with HCC.

C. Zhang and C. Zhang contributed equally to this work. 


\section{Cellular Physiology Cell Physiol Biochem 2018;51:289-300

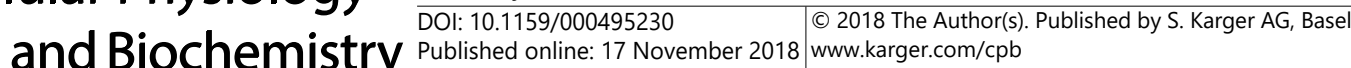 Zhang et al.: Circ_0091579 is a Diagnostic and Prognostic Marker for HCC}

\section{Introduction}

Hepatocellular carcinoma (HCC) is the fifth most prevalent malignant tumor and ranks as the second most common cause of cancer-related death worldwide [1,2]. Although the treatment and survival rate of patients with HCC has improved, most patients experience metastases and relapse within 5 years $[3,4]$. Since HCC is a heterogeneous disease with the accumulation of genetic and epigenetic changes $[5,6]$, it is critical to identify molecular biomarkers that can be used for its diagnosis and prognosis.

Circular RNAs (circRNAs), a unique form of RNA, possess covalently closed continuous loops without free ends; therefore, they are considerably more stable than linear RNAs [7-10]. Accumulating evidence has revealed that circRNAs can act as microRNA (miRNA) "sponges" and regulate RNA-binding proteins to control the expression of genes encoding proteins [11-14]. A growing number of studies have shown that the dysregulation of circRNA expression could have potential relationships with various human diseases such as systemic lupus erythematosus, diabetes mellitus, and Alzheimer's disease [15-17]. Recent studies have also revealed that circRNAs are involved in tumorigenesis, such as colon, gastric, and cervical cancer [18-21]. However, the use of circRNAs for the diagnosis and prognosis of patients with HCC remains largely unknown. Recently, some studies have centered on the role of circRNAs in affecting the survival of patients with HCC [22-23]. For instance, one study found that hsa_circ_0064428 was negatively correlated with the survival, tumor size, and metastasis of patients with HCC [24]. Besides, hsa_circ_0001445 has been reported to regulate the proliferation and migration of HCC and can serve as a diagnostic biomarker [25]. Furthermore, the oncogenic role of hsa_circ_0005075 has been confirmed since it was shown to promote HCC progression via the regulation of miR-431 [26]. Therefore, further screening and investigation of circRNAs in HCC are warranted.

In this study, we identified the differential expression profiles of circRNAs in three pairs of HCC clinical specimens with cancer thrombus using a circRNA microarray. We detected a total of 20 circRNAs with significantly different expression between tumor and non-tumor tissues. The altered expression of these circRNAs was verified using quantitative reverse transcription-polymerase chain reaction (qRT-PCR) analysis. We verified and validated the increased expression of hsa_circ_0091579 in patients with HCC. Then, we showed the role of hsa_circ_0091579 in the prognosis of survival in a cohort of patients with HCC. In conclusion, our findings suggested that hsa_circ_0091579 may serve as a promising biomarker for the prognosis of patients with HCC.

\section{Materials and Methods}

\section{Patients and samples}

A total of 105 HCC and paired adjacent non-cancerous tissue samples (30 in the training set and 75 in the validation set) were obtained from Fudan University Shanghai Cancer Center. The participants were recruited in January 2011. The samples were collected between January 1, 2011 and December 31, 2015. We conducted this study in January 2018. All patients included in this study gave their written informed consent prior to surgery. The study conformed with the code of ethics of the World Medical Association (Declaration of Helsinki). The samples were snap-frozen immediately in liquid nitrogen after resection and stored at $-80^{\circ} \mathrm{C}$ until RNA extraction. The diagnosis of HCC was confirmed pathologically. Three pairs of the samples were applied for microarray analysis, and 30 pairs were collected for qRT-PCR validation. We defined overall survival (OS) as the duration between diagnosis and death or between diagnosis and the last observation point. For surviving patients, the data were censored at the last follow-up. The use of human samples was approved by the Ethics Committee of Shangdong Cancer Hospital. We had access to personally identifying information of individual participants during and after data collection. 


\section{Cellular Physiology Cell Physiol Biochem 2018;51:289-300

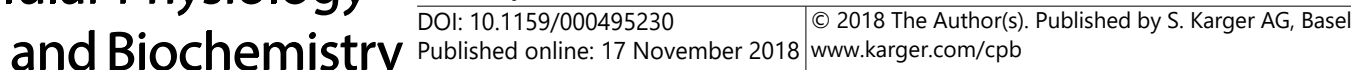 Zhang et al.: Circ_0091579 is a Diagnostic and Prognostic Marker for HCC}

\section{RNA extraction and $q R T-P C R$}

Total RNA was extracted from the HCC tissue samples and matched adjacent non-tumor tissues using the TRIzol reagent (Invitrogen, Karlsruhe, Germany) according to the manufacturer's instructions. cDNA was synthesized with PrimeScript RT reagents (Takara Bio, Inc., Kusatsu, Japan). Primers for specific genes were designed and synthesized by Thinkgene Co. (Shanghai, China). The relative expression of circRNA was performed on an ABI Prism 7900 sequence detection system (Applied Biosystems, Foster City, CA). CircRNA expression was normalized to the expression of the housekeeping gene GAPDH.

\section{CircRNA microarray hybridization and expression profiling}

Microarray profiling was conducted by CapitalBio Technology Corporation (Beijing, China) using a human $8 \times 15 \mathrm{~K}$ CIRCRNA Array (Arraystar Technologies, Rockville, MD). Sample labeling, microarray hybridization, and washing were performed according to the manufacturer's standard protocols. Briefly, each purified RNA sample was transcribed to double-stranded cDNA, synthesized into cRNA, and labeled with Cy3-dCTP. The labeled cRNAs were hybridized with the CapitalBio Technology Human CircRNA Microarray. Next, the array was washed and scanned using an Agilent Scanner G2505C (KangChen Bio-tech, Shanghai, China). Quantile normalization of the raw data and subsequent data processing were performed using the R software package (R Version 3.1.2). CircRNAs with a fold change in expression $\geq 2.0$ and $P$-value $<0.05$ between the tumor and adjacent non-tumor tissues were considered statistically significant.

Primers for quantitative amplification of circRNAs

The sequences of these primers are listed in Table 1.

\section{$X$-tile analysis}

X-tile software was used to determine the optimal cut-off value of hsa_circ_0091579 expression according to the user's manual and previous studies [27-28].

\section{Statistical analysis}

Student's t-test was used to analyze differences in the expression of circRNAs between HCC and adjacent non-cancerous tissues. Spearman's rank correlation coefficient was utilized to calculate bivariate correlations. Receiver operating characteristic (ROC) curves were established to evaluate the diagnostic value of hsa_circ_0091579 and hsa_circ_16245-1. The Youden index, also known as the correct index, was applied to evaluate the authenticity of the screening test. Survival analysis was performed using the Kaplan-Meier method. Cox regression analyses were also performed to assess the association between hsa circ_0091579 and OS of HCC patients. The above statistical analyses were performed by using SPSS software package (version 22.0; SPSS, Inc., Chicago, IL) and GraphPad Prism 6.0 (GraphPad Software, La Jolla, CA). X-tile software (version 3.5.0) was applied to determine the cut-off value of hsa_circ_0091579 expression. A $P$-value less than 0.05 was considered to be statistically significant.

Table 1. Primers for qRT-PCR

\begin{tabular}{lcc}
\hline Name & Forward Primer $\left(5^{\prime}-3^{\prime}\right)$ & Reverse Primer $\left(5^{\prime}-3^{\prime}\right)$ \\
\hline hsa_circ_0091579 & TGAGCCAGTGGTCAGTCAAA & GTGGAGTCAGGCTTGGGTAG \\
hsa_circ_15058-21 & GAGAGCTGTGAGCAAGTGAA & GAGGCGGGATATTTCGTATCTG \\
hsa_circ_3424-4 & AAAGAGGCTAAGGACACCAAA & TCAGGTCCCTGTGCTATCT \\
hsa_circ_16245-1 & GAAACCTTATCCAGCCGAAGA & GTCAGGCTTGGGTAGTTGTT \\
GAPDH & TCGACAGTCAGCCGCATCTTCTTT & ACCAAATCCGTTGACTCCGACCTT \\
\hline
\end{tabular}




\section{Cellular Physiology Cell Physiol Biochem 2018;51:289-300 and Biochemistry \begin{tabular}{l|l} 
DOI: 10.1159/000495230 2018 The Author(s). Published by S. Karger AG, Basel \\
Publishedon 2018
\end{tabular}

\section{Results}

\section{Deregulated circRNAs in HCC tumor tissues}

To investigate circRNA expression profiles in HCC tissues, we analyzed three pairs of HCC tissue samples (three HCC tissues and three matched non-tumor liver tissues) by using a circRNA microarray. Through expression intensity sorting within the HCC tumor and nontumor groups, the 20 most increased circRNAs in HCC tumor tissues as compared with those in non-tumor tissues are shown in Fig. 1A.

\section{Validation of differentially expressed circRNAs by qRT-PCR}

Among the 20 differentially expressed circRNAs, five circRNAs that were most highly expressed in HCC tissues were selected for validation using tumor and non-tumor tissues from 30 HCC patients by qRT-PCR. The expression of hsa_circ_15058-17 was not detected by qRT-PCR. As presented in Fig. 2, the expression of hsa_circ_16245-1 and hsa_circ_0091579 was consistently and significantly increased in HCC tumor tissues, while there was no dramatic difference in the levels of hsa_circ_3424-4 and hsa_circ_15058-21 between HCC and paired adjacent non-tumor tissues.

Potential diagnostic value of hsa_circ_16245-1 and hsa_circ_0091579 in HCC

Since we found that hsa_circ_16245-1 and hsa_circ_0091579 levels were significantly increased in HCC tissues, we evaluated their potential diagnostic value. ROC curves were generated for hsa_circ_16245-1 and hsa_circ_0091579 levels in HCC. We found that the area under the ROC curve was 0.656 for hsa_circ_0091579 and 0.720 for hsa_circ_16245-1 (Fig. $3)$. The sensitivity and specificity of hsa_circ_0091579 was 0.97 and 0.4 , respectively. The sensitivity and specificity of hsa_circ_16245-1 was 0.83 and 0.63 , respectively. To validate their prognostic value further, a total of 75 pairs of HCC samples were employed in a validation group. Hsa_circ_0091579 expression was found to be significantly increased in HCC tumor tissue as compared with that in adjacent non-tumor tissue; however, hsa_circ_16245-1 was not detected in some of the HCC samples (Fig. 4). In addition, the sensitivity and specificity of hsa_circ_0091579 for diagnosis were also confirmed (Fig. 4).

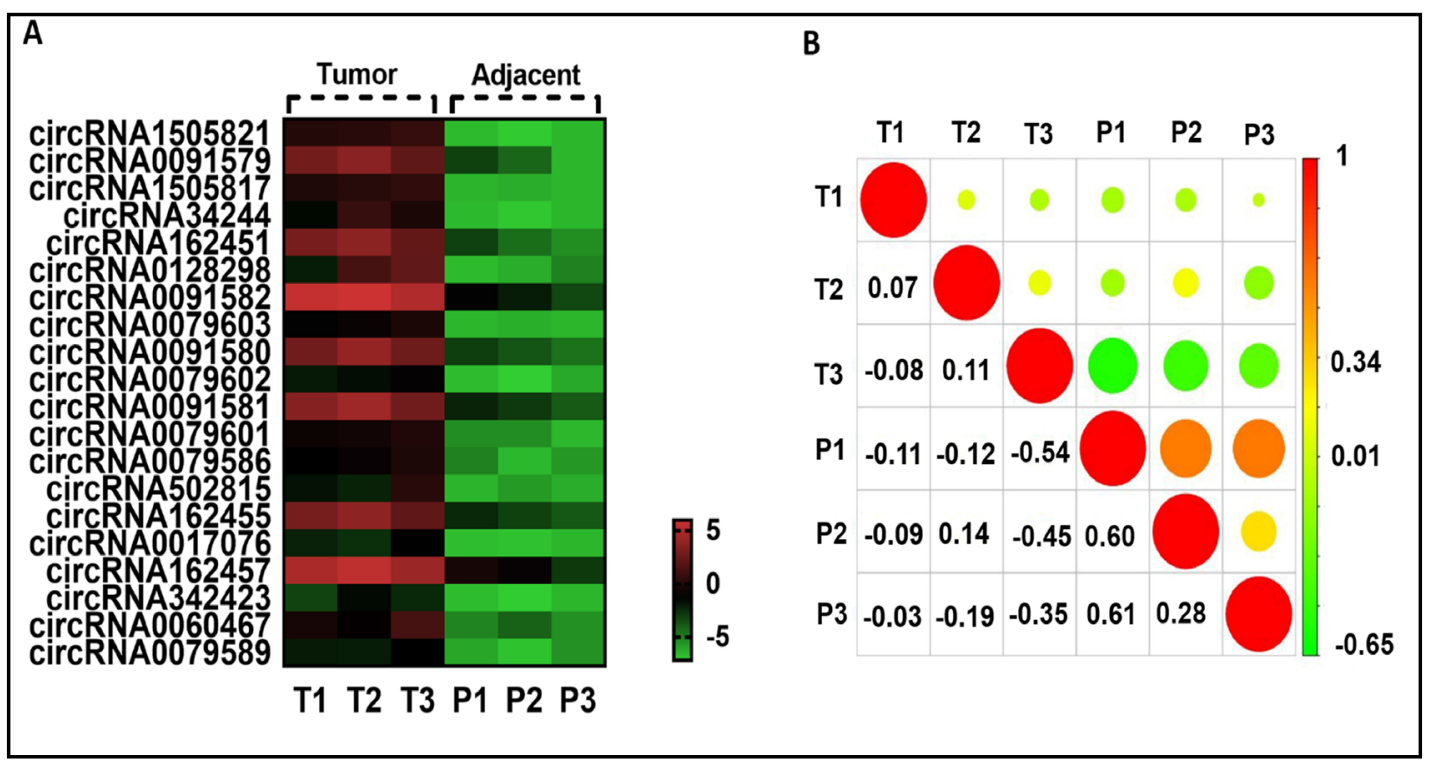

Fig. 1. Deregulated circRNAs in HCC tumor tissues. (A) Heat map showing the top 20 most increased circRNAs in HCC tissues as compared with matched paratumor tissues analyzed by a circRNA microarray. (B) Differences of gene expression between tumor and adjacent non-tumor tissues.

\section{KARGER}




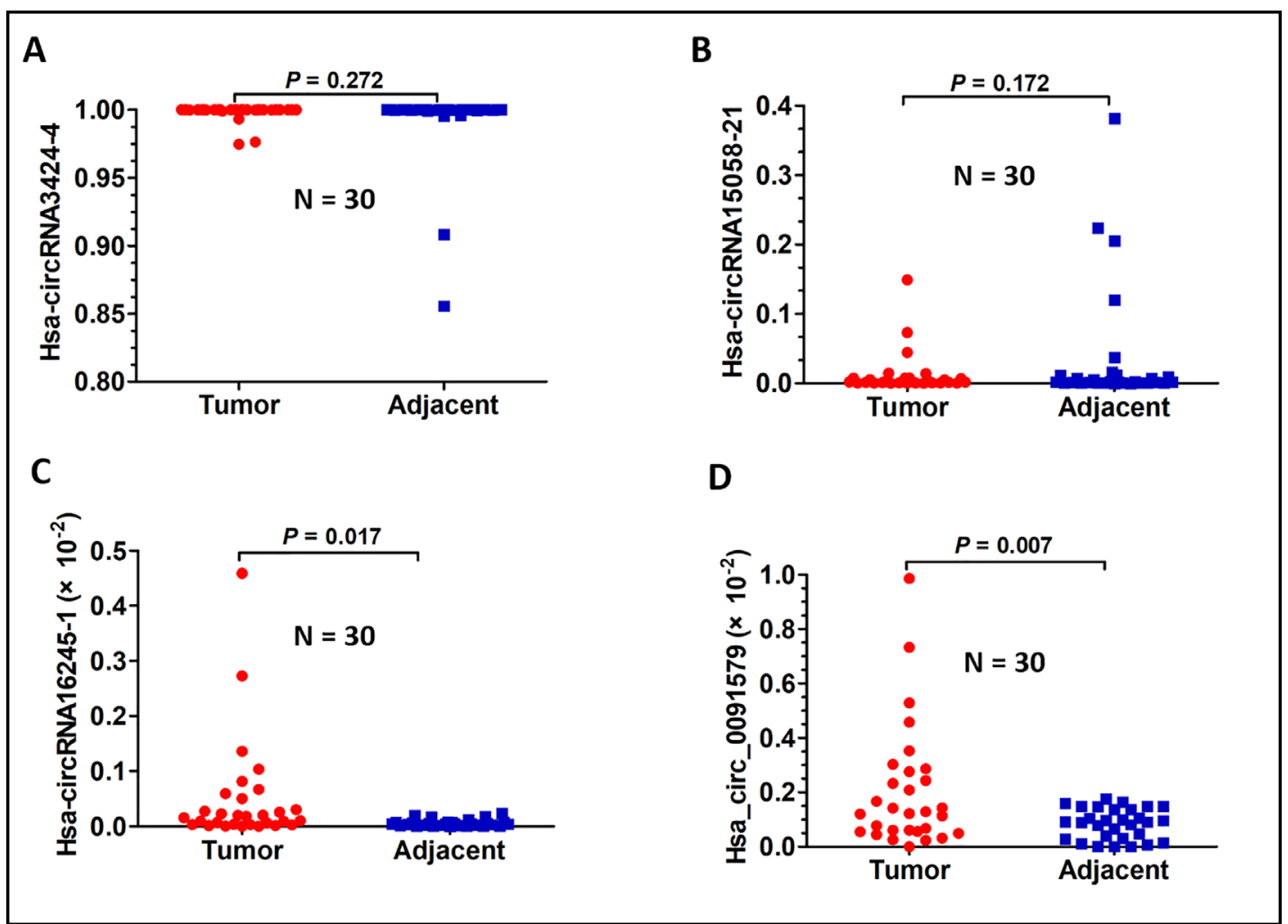

Fig. 2. Increased hsa_circ_16245-1 and hsa_circ_0091579 expression in HCC tissues. Among the 20 differentially expressed circRNAs, five circRNAs with the most increased expression in HCC tissues were chosen. We tested their expression in tumor and non-tumor tissues from 30 HCC patients by qRT-PCR. The expression of hsa_circ_15058-17 was not detected by qRT-PCR.

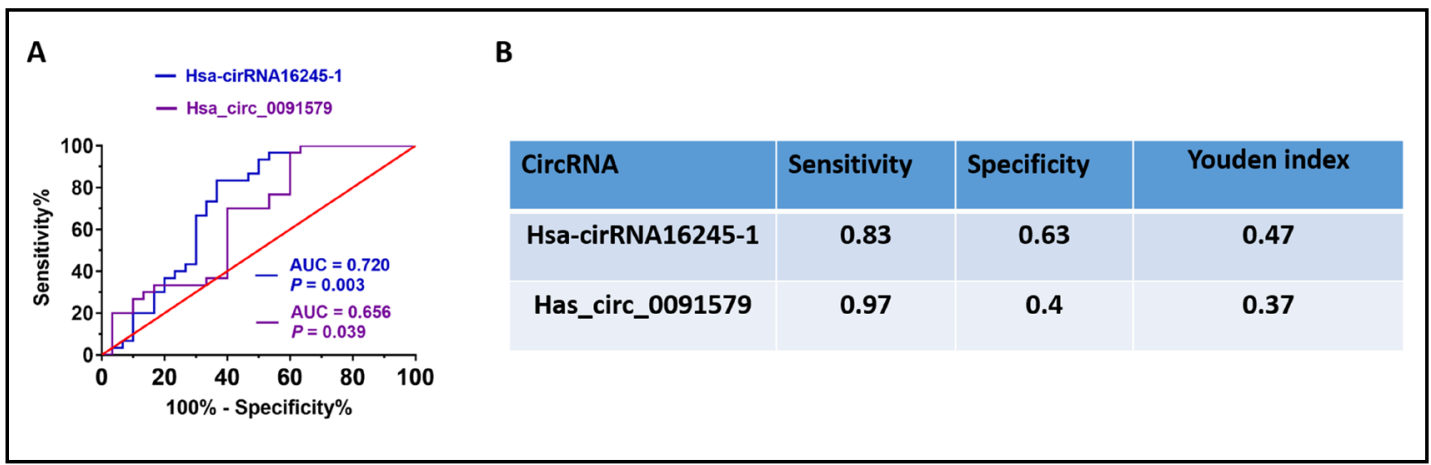

Fig. 3. Diagnostic value of hsa_circ_16245-1 and hsa_circ_0091579 in HCC. (A) Receiver operation characteristic curves of hsa_circ_16245-1 and hsa_circ_0091579 as markers to differentiate HCC. (B) Sensitivity, specificity, and the Youden index of hsa_circ_16245-1 and hsa_circ_0091579 in the diagnosis of HCC.

Increased hsa_circ_0091579 expression is significantly correlated with poor prognosis in HCC patients

As hsa_circ_0091579 expression was significantly increased in HCC tissues, we next analyzed whether its increased expression correlated with the prognosis of patients with HCC. A cohort of HCC patients with survival data was included, and hsa_circ_0091579 expression was determined using in situ hybridization analysis of tissue array slides. Increased hsa_circ_0091579 expression in HCC tissues was significantly correlated with the 


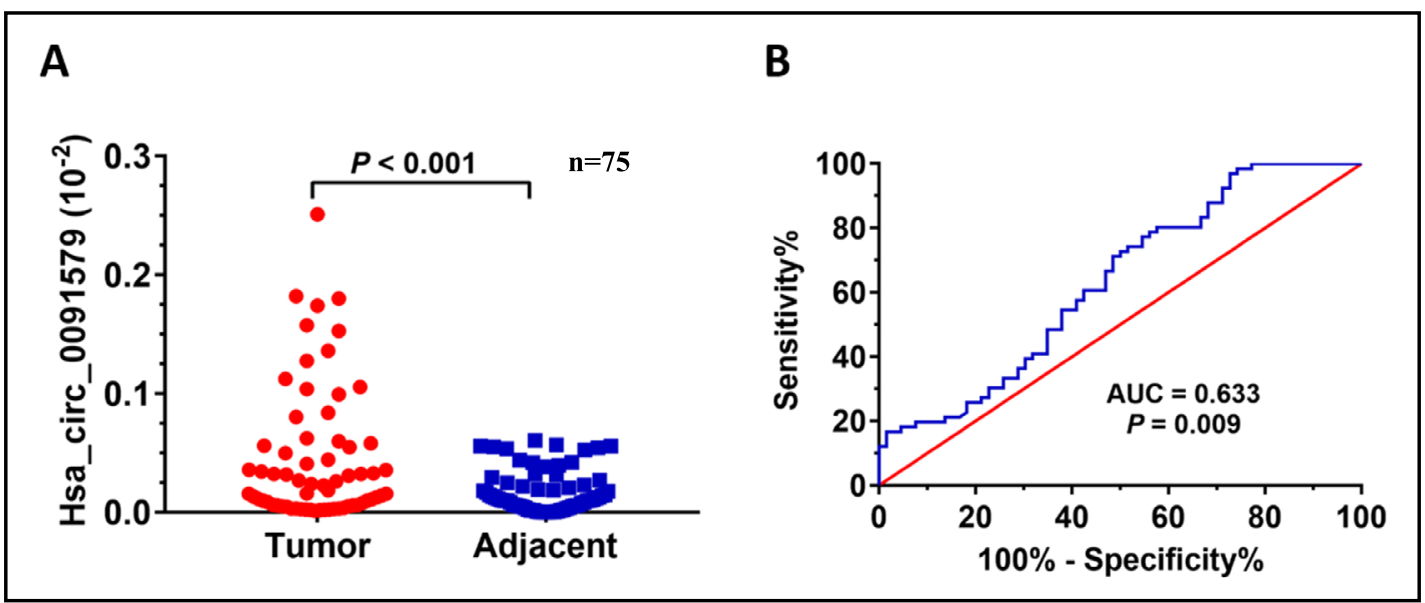

Fig. 4. Reassessment of the diagnostic value of hsa_circ_0091579 in HCC. (A) Differential expression and statistical analysis of hsa_circ_0091579 between HCC and matched adjacent non-tumor tissues was validated in an additional 75 patients by qRT-PCR. (B) Receiver operation characteristic curve of hsa_circ_0091579 as a marker to differentiate HCC.

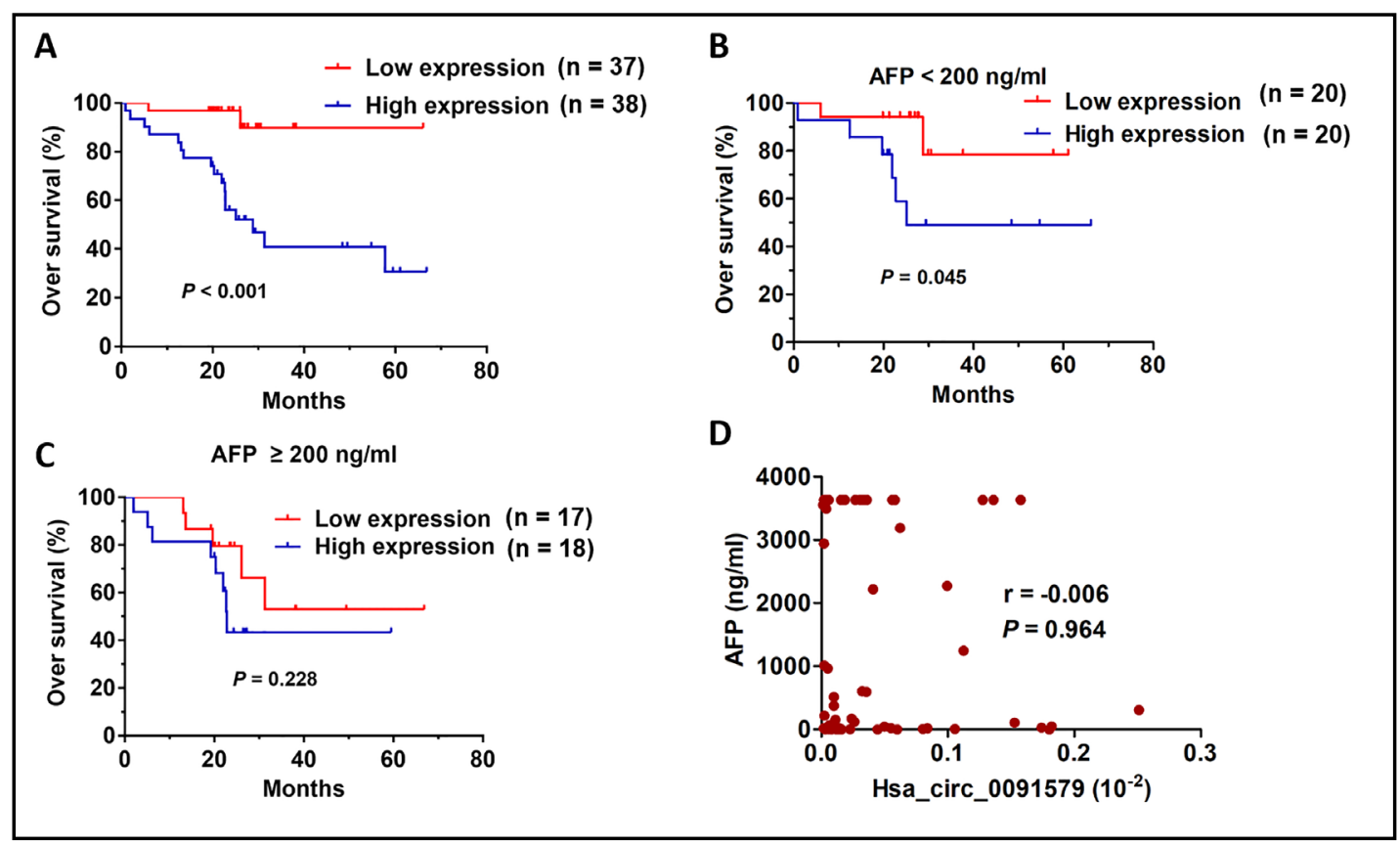

Fig. 5. Hsa_circ_0091579 expression in HCC tissues correlates with the prognosis of patients with HCC. (A) Prognostic significance of hsa_circ_0091579 expression for HCC patients was performed by using the median value as the cut-off, and the observation time was 140 months. (B) and (C) Prognostic significance of hsa_circ_0091579 expression for HCC patients was performed by using the median value as the cut-off in HCC patients with AFP $<200 \mathrm{ng} / \mathrm{mL}$ (B) and AFP $\geq 200 \mathrm{ng} / \mathrm{mL}$ (C). (D) The association between AFP and hsa_circ_0091579 expression was evaluated by Spearman's rank correlation coefficient.

poor prognosis of HCC patients (Fig. 5), shown as a Kaplan-Meier survival curve using the median value of hsa_circ_0091579 expression as the cut-off. Further correlation analysis demonstrated that there was no association between the levels of alpha fetoprotein (AFP) and hsa_circ_0091579. 


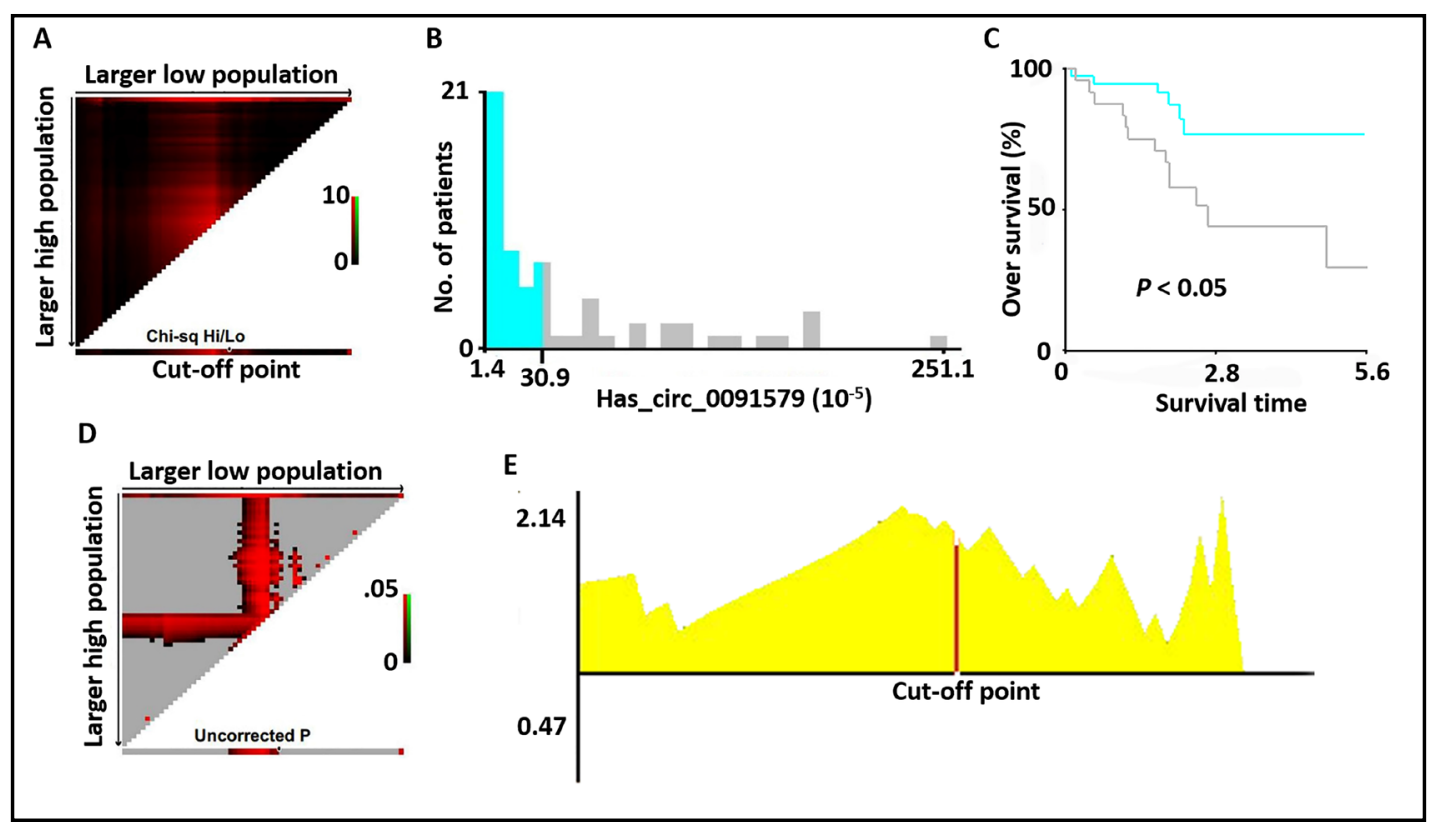

Fig. 6. Validation of hsa_circ_0091579 expression in HCC tissues and its correlation with the prognosis of patients with HCC. (A) X-tile plots based on the expression of hsa_circ_0091579. The data on the horizontal ordinate increase from the left to the right, defined as the larger low population. The data on the vertical ordinate decrease from the top to the bottom, defined as the larger high population. (B) and (C) Prognostic significance of hsa_circ_0091579 expression for HCC patients was performed by using the statistical algorithm in X-tile software to calculate the most efficient cut-off value for hsa_circ_0091579 expression $\left(30.9 \times 10^{-5}\right)$. (D) The P-value of OS based on the optimal cut-off value. (E) Relative risk plot of HCC patients with high hsa_circ_0091579 expression compared with those with low expression.

\section{Cut-off value based on outcome and Kaplan-Meier analysis}

Furthermore, we used X-tile software to calculate the most efficient cut-off value $(30.9 \times$ $10^{-5}$ ) of hsa_circ_0091579 expression that could most significantly distinguish the outcome of patients with HCC patients (Fig. 6A, 6B). OS was further analyzed using Kaplan-Meier analysis through this software. The results showed that HCC patients with relatively low hsa_circ_0091579 expression had improved OS compared with those with high expression $(P<0.05$; Fig. 6C). The $P$-value of OS based on the optimal cut-off point and a relative risk plot of HCC patients with high hsa_circ_0091579 expression compared with those with low expression are shown in Fig. 6D and Fig. 6E, respectively.

Together, these data suggest that increased hsa_circ_0091579 expression in HCC is correlated with the poor prognosis of patients, and the level of hsa_circ_0091579 expression may be useful for the prognosis of patients with HCC.

\section{Discussion}

The morbidity of HCC has been on the rise during the past few decades, especially in Asian countries [1-2]. Thus, identifying markers for the diagnosis and prognosis of HCC is greatly needed.

CircRNAs were previously believed to possess no biological function [29-30]; however, both RNA deep sequencing technology and the development of bioinformatics have deepened our understanding of circRNAs. One study found that a series of circRNAs are aberrantly expressed in early-stage lung adenocarcinoma, which has made the early detection of lung cancer a possibility [31]. Another study revealed that a group of circRNAs specifically participate in the development of pancreatic ductal adenocarcinoma [32]. 


\section{Cellular Physiology Cell Physiol Biochem 2018;51:289-300

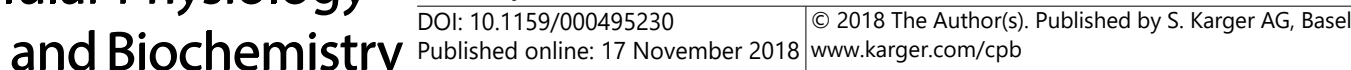 \\ Zhang et al.: Circ_0091579 is a Diagnostic and Prognostic Marker for HCC}

As the expression and function of circRNAs during the development of HCC are still largely elusive, we screened for circRNAs that were differentially expressed between HCC tissues with cancer thrombus and paired tissues using a chip microarray. Then, we examined the expression of the top 20 circRNAs identified by the microarray, after which two circRNAs were chosen for further analysis. Since hsa_circ_0091579 was found to be more accurate than hsa_circ_16245-1 for prognosis, we focused on this circRNA for additional analysis.

Hsa_circ_0091579 expression was significantly increased in HCC and linked with an unfavorable prognosis in patients with HCC. By using X-tile software, we obtained an efficient cut-off value of $30.9 \times 10^{-5}$ for hsa_circ_0091579 to distinguish the outcome of patients with HCC. Although the exact applicable cut-off value still needs to be confirmed in a large set of patients, this cut-off value for hsa_circ_0091579 is somewhat practical for the prognosis of HCC patients. However, the increased expression of hsa_circ_0091579 was not associated with serum AFP level, tumor diameter, differentiation, Barcelona Clinic Liver Cancer stage, and tumor-node-metastasis stage in HCC in our study (data not shown). This may be due to the complex tumor microenvironment and the relatively small sample size examined. Currently, a series of circRNAs, e.g., hsa_circ_0005075, hsa_circ_0004018, and hsa_circ_0003570, have been demonstrated to influence the development and prognosis of HCC [33-35]. However, conclusive or practical criteria for the prognosis of HCC still require further investigation.

In circBase, which is a database for circRNAs, it has been shown that hsa_circ_0091579 regulates the glypican-3 protein (GPC3) gene. GPC3 is a member of the heparan sulfate proteoglycan family. It is normally detected in placenta, numerous embryonic tissues, adult ovary, mammary gland, mesothelium, lung, and kidney, while no GPC3 expression can be detected in healthy adult liver [36-37]. Previous studies have demonstrated a dramatic increase in GPC3 levels in both serum and tumor tissue in HCC [38]. Multiple mechanisms may account for the involvement of GPC3 in the progression of HCC, including Wnt signaling activation, macrophage recruitment, cell growth, and the promotion of epithelial to mesenchymal transition [39-43]. It is also noteworthy that by sequestering miRNAs, circRNAs are major players in the miRNA-mediated regulation of corresponding mRNA and gene expression, and are therefore closely related to the development of various human cancers. For example, the circRNA ciRS-7/CRD1 as contains multiple, tandem miR-7 binding sites, and, thereby, acts as an endogenous miRNA sponge to adsorb, and hence quench, the normal functions of miR-7 [44-45]. To the best of our knowledge, four miRNAs (miR-96, miR-1271, miR-520c-3p, and miR-219-5p) have been shown to regulate GPC3 expression negatively [46-49]. Therefore, we speculate that hsa_circ_0091579 might competitively bind with miR-96, miR-1271, miR-520c-3p, and miR-219-5p and abrogate their effects. The interactions between hsa_circ_0091579 and the above miRNAs remain largely unknown and require further research.

As reported previously, some non-coding RNAs are stable in plasma, while some circRNAs are abundant in exosomes [50-51]. Whether hsa_circ_0091579 can also be detected in the plasma or even in circulating exosomes and whether circulating hsa_circ_0091579 is also associated with HCC development and prognosis are critical questions that require further investigation in the future.

The findings of this study could be utilized in clinical practice for the diagnosis and prognosis of patients with HCC. Prognosis could be largely determined by the detection of hsa_circ_0091579 in tumors from HCC patients. Therefore, different treatments could be designed for HCC patients with different expression levels of hsa_circ_0091579. Thus, individual-oriented therapy could be achieved. 


\section{Cellular Physiology Cell Physiol Biochem 2018;51:289-300

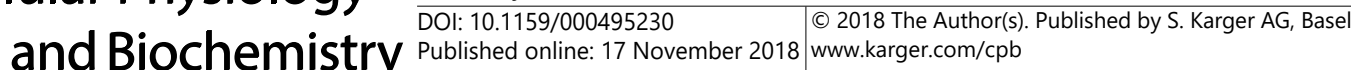

\section{Conclusion}

To conclude, our study revealed the overexpression of hsa_circ_0091579 in HCC tissues as compared with their adjacent non-tumor tissues, and increased hsa_circ_0091579 expression was linked with worse OS in patients with HCC. However, a relatively larger study should be performed to confirm our findings further so that hsa_circ_0091579 expression can be applied in the clinical setting in the future.

\section{Acknowledgements}

This study was supported jointly by the National Natural Science Foundation of China (No. 81603348); China Postdoctoral Fund (No. 21300075311104); Shandong Postdoctoral Innovation Special Fund (No. 201602012); China Postdoctoral Special Fund (No. 2018T110696), Shandong Province Key R \& D Plan (No. 2018GSF119014) and CSCO - Lizhu Chinese Medicine Cancer Research Fund (Y-L2018-009).

\section{Disclosure Statement}

The authors have no conflicts of interest to declare.

\section{References}

$>1$ Torre LA, Bray F, Siegel RL, Ferlay J, Lortet-Tieulent J, Jemal A: Global cancer statistics, 2012. CA Cancer J Clin 2015;65:87-108.

-2 Ferlay J, Soerjomataram I, Dikshit R, Eser S, Mathers C, Rebelo M, Parkin DM, Forman D, Bray F: Cancer incidence and mortality worldwide: Sources, methods and major patterns in GLOBOCAN 2012. Int J Cancer 2015;136:E359-E386.

3 Xing X, Liang D, Huang Y, Zeng Y, Han X, Liu X, Liu J: The application of proteomics in different aspects of hepatocellular carcinoma research. J Proteomics 2016;145:70-80.

4 Tsai TH, Song E, Zhu R, Di Poto C, Wang M, Luo Y, Varghese RS, Tadesse MG, Ziada DH, Desai CS, Shetty K, Mechref Y, Ressom HW: LC-MS/MS-based serum proteomics for identification of candidate biomarkers for hepatocellular carcinoma. Proteomics 2015;15:2369-2381.

5 Zhao Y, Li Y, Ma H, Dong W, Zhou H, Song X, Zhang J, Jia L: Modification of sialylation mediates the invasive properties and chemosensitivity of human hepatocellular carcinoma. Mol Cell Proteomics 2014;13:520536.

6 Megger DA, Bracht T, Kohl M, Ahrens M, Naboulsi W, Weber F, Hoffmann AC, Stephan C, Kuhlmann K, Eisenacher M, Schlaak JF, Baba HA, Meyer HE, Sitek B: Proteomic differences between hepatocellular carcinoma and nontumorous liver tissue investigated by a combined gel-based and label-free quantitative proteomics study. Mol Cell Proteomics 2013;12:2006-2020. Wilusz JE, Sharp PA: Molecular biology. A circuitous route to noncoding RNA. Science 2013;340:440-441. Chen LL, Yang L: Regulation of circRNA biogenesis. RNA Biol 2015; 12: 381-388. Lasda E, Parker R: Circular RNAs: diversity of form and function. RNA 2014;20:1829-1842. Vicens Q, Westhof E: Biogenesis of circular RNAs. Cell 2014;159:13-14. Hansen TB, Jensen TI, Clausen BH, Bramsen JB, Finsen B, Damgaard CK, Kjems J: Natural RNA circles function as efficient microRNA sponges. Nature 2013; 495: 384-388.

12 Guo JU, Agarwal V, Guo H, Bartel DP: Expanded identification and characterization of mammalian circular RNAs. Genome Biol 2014;15:409.

-13 Ashwal-Fluss R, Meyer M, Pamudurti NR, Ivanov A, Bartok O, Hanan M, Evantal N, Memczak S, Rajewsky N, Kadener S: CircRNA biogenesis competes with pre-mRNA splicing. Mol Cell 2014;56:55-66.

14 Rong D, Sun H, Li Z, Liu S, Dong C, Fu K, Tang W, Cao H: An emerging function of circRNA-miRNAs-mRNA axis in human disease. Oncotarget 2017;8:73271-73281. 


\section{Cellular Physiology Cell Physiol Biochem 2018;51:289-300

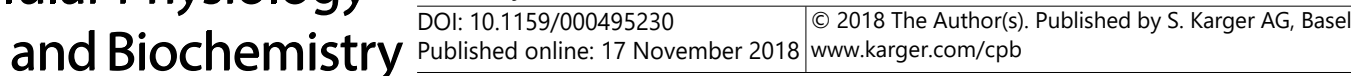 \\ Zhang et al.: Circ_0091579 is a Diagnostic and Prognostic Marker for HCC}

15 Zhao Y, Alexandrov PN, Jaber V, Lukiw WJ: Deficiency in the ubiquitin conjugating enzyme UBE2A in Alzheimer's disease (AD) is linked to deficits in a natural circular miRNA-7 sponge (circRNA; ciRS-7). Genes (Basel) 2016; 7:E116.

16 Li C, Zhao L, Jiang W, Che L, Xu Y: Correct microarray analysis approaches in Hsa-circRNA11783-2 in peripheral blood is correlated with coronary artery disease and type 2 diabetes mellitus. Diab Vasc Dis Res 2018;15:92-93.

17 Li H, Li K, Lai W, Li X, Wang H, Yang J, Chu S, Wang H, Kang C, Qiu Y: Comprehensive circular RNA profiles in plasma reveals that circular RNAs can be used as novel biomarkers for systemic lupus erythematosus. Clin Chim Acta 2018;480:17-25.

18 Gao YL, Zhang MY, Xu B, Han LJ, Lan SF, Chen J, Dong YJ, Cao LL: Circular RNA expression profiles reveal that hsa_circ_0018289 is up-regulated in cervical cancer and promotes the tumorigenesis. Oncotarget 2017;8:86625-86633.

19 Huang M, He YR, Liang LC, Huang Q Zhu ZQ: Circular RNA hsa_circ_0000745 may serve as a diagnostic marker for gastric cancer. World J Gastroenterol 2017;23:6330-6338.

20 Hsiao KY, Lin YC, Gupta SK, Chang N, Yen L, Sun HS, Tsai SJ: Noncoding Effects of Circular RNA CCDC66 Promote Colon Cancer Growth and Metastasis. Cancer Res 2017;77:2339-2350.

21 Weng W, Wei Q, Toden S, Yoshida K, Nagasaka T, Fujiwara T, Cai S, Qin H, Ma Y, Goel A: Circular RNA ciRS-7-A Promising Prognostic Biomarker and a Potential Therapeutic Target in Colorectal Cancer. Clin Cancer Res 2017;23:3918-3928.

22 Qiu LP, Wu YH, Yu XF, Tang Q Chen L, Chen KP: The Emerging Role of Circular RNAs in Hepatocellular Carcinoma. J Cancer 2018;9:1548-1559.

23 Fu L, Jiang Z, Li T, Hu Y, Guo J: Circular RNAs in hepatocellular carcinoma: Functions and implications. Cancer Med 2018;doi:10.1002/cam4.1574.

-24 Weng Q Chen M, Li M, Zheng YF, Shao G, Fan W, Xu XM, Ji J: Global microarray profiling identified hsa circ_0064428 as a potential immune-associated prognosis biomarker for hepatocellular carcinoma. J Med Genet 2018;doi:10.1136/jmedgenet-2018-105440.

-25 Zhang X, Zhou H, Jing W, Luo P, Qiu S, Liu X, Zhu M, Liang C, Yu M, Tu J: The Circular RNA hsa_circ_0001445 regulates the proliferation and migration of hepatocellular carcinoma and may serve as a diagnostic biomarker. Dis Markers 2018;2018:3073467.

-26 Li MF, Li YH, He YH, Wang Q, Zhang Y, Li XF, Meng XM, Huang C, Li J: Emerging roles of hsa_circ_0005075 targeting miR-431 in the progress of HCC. Biomed Pharmacother 2018;99:848-858.

27 Altman DG, Lausen B, Sauerbrei W, Schumacher M: Dangers of using "optimal” cutpoints in the evaluation of prognostic factors. J Natl Cancer Inst 1994;86:829-835.

28 Altman DG: Suboptimal analysis using 'optimal' cutpoints. Br J Cancer 1998;78:556-557.

29 Hsu MT, Coca-Prados M: Electron microscopic evidence for the circular form of RNA in the cytoplasm of eukaryotic cells. Nature. 1979;280:339-340.

-30 Arnberg AC, Van Ommen GJ, Grivell LA, Van Bruggen EF, Borst P: Some yeast mitochondrial RNAs are circular. Cell 1980;19:313-319.

-31 Zhao J, Li L, Wang Q, Han H, Zhan Q, Xu M: CircRNA Expression Profile in Early-Stage Lung Adenocarcinoma Patients. Cell Physiol Biochem 2017;44:2138-2146.

-32 Li H, Hao X, Wang H, Liu Z, He Y, Pu M, Zhang H, Yu H, Duan J, Qu S: Circular RNA expression profile of pancreatic ductal adenocarcinoma revealed by microarray. Cell Physiol Biochem 2016;40:1334-1344.

-33 Shang X, Li G, Liu H, Li T, Liu J, Zhao Q Wang C: Comprehensive Circular RNA profiling reveals that hsa_circ_0005075, a new circular RNA biomarker, is involved in hepatocellular carcinoma development. Medicine (Baltimore) 2016;95:e3811.

34 Fu L, Yao T, Chen Q, Mo X, Hu Y, Guo J: Screening differential circular RNA expression profiles reveals hsa_ circ_0004018 is associated with hepatocellular carcinoma. Oncotarget 2017;8:58405-58416.

35 Fu L, Wu S, Yao T, Chen Q, Xie Y, Ying S, Chen Z, Xiao B, Hu Y: Decreased expression of hsa_circ_0003570 in hepatocellular carcinoma and its clinical significance. J Clin Lab Anal 2018;32.doi:10.1002/jcla.22239.

-36 Iglesias BV, Centeno G, Pascuccelli H, Ward F, Peters MG, Filmus J, Puricelli L, de Kier Joffé EB: Expression pattern of glypican-3 (GPC3) during human embryonic and fetal development. Histol Histopathol 2008;23:1333-1340.

-37 Filmus J: Glypicans in growth control and cancer. Glycobiology 2001; 11:19R-23R. 


\section{Cellular Physiology Cell Physiol Biochem 2018;51:289-300

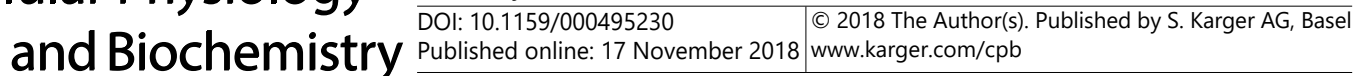

Zhang et al.: Circ_0091579 is a Diagnostic and Prognostic Marker for HCC

-38 Capurro M, Wanless IR, Sherman M, Deboer G, Shi W, Miyoshi E, Filmus J: Glypican-3: A novel serum and histochemical marker for hepatocellular carcinoma. Gastroenterology 2003;125:89-97.

-39 Capurro MI, ShiW, Sandal S, Filmus J: Processing by convertases is not required for glypican-3-induced stimulation of hepatocellular carcinoma growth. J Biol Chem 2005;280:41201-41206.

$>40$ Capurro MI, Xiang YY, Lobe C, Filmus J: Glypican-3 promotes the growth of hepatocellular carcinoma by stimulating canonical Wnt signaling. Cancer Res 2005;65:6245-6254.

-41 Midorikawa Y, Ishikawa S, Iwanari H, Imamura T, Sakamoto H, Miyazono K, Kodama T, Makuuchi M, Aburatani H: Glypican-3, overexpressed in hepatocellular carcinoma, modulates FGF2 and BMP-7 signaling. Int J Cancer 2003;103:455-465.

42 Takai H, Ashihara M, Ishiguro T, Terashima H, Watanabe T, Kato A, Suzuki M: Involvement of glypican-3 in the recruitment of M2-polarized tumor-associated macrophages in hepatocellular carcinoma. Cancer Biol Ther 2009;8:2329-2338.

43 Wu Y, Liu H, Weng H, Zhang X, Li P, Fan CL, Li B, Dong PL, Li L, Dooley S, Ding HG: Glypican-3 promotes epithelial mesenchymal transition of hepatocellular carcinoma cells through ERK signaling pathway. Int J Oncol 2015;46:1275-1285.

-44 Peng L, Yuan XQ Li GC: The emerging landscape of circular RNA ciRS-7 in cancer (Review). Oncol Rep 2015;33:2669-2674.

45 Hansen, TB, Kjems J, Damgaard, CK: Circular RNA and miR-7 in cancer. Cancer Res 2013;73:5609-5612.

-46 Maurel M, Jalvy S, Ladeiro Y, Combe C, Vachet L, Sagliocco F, Bioulac-Sage P, Pitard V, Jacquemin-Sablon H, Zucman-Rossi J, Laloo B, Grosset CF: A functional screening identifies five microRNAs controlling glypican-3: Role of miR-1271 down-regulation in hepatocellular carcinoma. Hepatology 2013;57:195-204.

-47 Jalvy-Delvaille S, Maurel M, Majo V, Pierre N, Chabas S, Combe C, Rosenbaum J, Sagliocco F, Grosset CF: Molecular basis of differential target regulation by miR-96 and miR-182: The glypican-3 as a model. Nucleic Acids Res 2012;40:1356-1365.

48 Miao HL, Lei CJ, Qiu ZD, Liu ZK, Li R, Bao ST, Li MY: MicroRNA-520c-3p inhibits hepatocellular carcinoma cell proliferation and invasion through induction of cell apoptosis by targeting glypican-3. Hepatol Res 2014;44:338-348.

49 Huang N, Lin J, Ruan J, Su N, Qing R, Liu F, He B, Lv C, Zheng D, Luo R: MiR-219-5p inhibits hepatocellular carcinoma cell proliferation by targeting glypican-3. FEBS Lett 2012;586:884-891.

50 Yuan SX, Wang J, Yang F, Tao QF, Zhang J, Wang LL, Yang Y, Liu H, Wang ZG, Xu QG, Fan J, Liu L, Sun SH, Zhou WP: Long noncoding RNA DANCR increases stemness features of hepatocellular carcinoma by derepression of CTNNB1. Hepatology 2016;63:499-511.

51 Li Y, Zheng Q, Bao C, Li S, Guo W, Zhao J, Chen D, Gu J, He X, Huang S: Circular RNA is enriched and stable in exosomes: a promising biomarker for cancer diagnosis. Cell Res 2015;25:981-984. 